\title{
Subsidized Tick-Borne ENCEPHALITIS-VACCINATION to CHILDREN IN STOCKHOLM COUNTY, SWEdeN - A HEALTH ECONOMIC PERSPECTIVE
}

\section{Jad Shedrawy*, Martin Henriksson Martin, Maria-Pia Hergens Maria-Pia, Helena Hervius Askling}

\section{Introduction}

the number of notified cases of TickBorne Encephalitis (TBE) in sweden has been increasing the past years despite the increased use of TBEvaccine which is not subsidized by the healthcare system.

Stockholm County is a high endemic area and an earlier study has shown that low-income households have lower vaccination coverage even when they are at high risk.

\section{Aim}

Determine the cost-effectiveness of a publicly funded TBE vaccination program in Stockholm.

\section{Methods}

In three different cohorts with individuals aged 3,40 or 50 years, long-term costs and health outcomes were modeled through a Markov model with two arms of comparison:

- no vaccination

- vaccination program

The Markov model predicts the costs and effects (in term of Qualityadjusted Life Years (QALYs) over a lifetime horizon using a healthcare perspective.

\section{Results}

The primary results are presented as an incremental cost effectiveness ratio (ICER) which is the cost required to achieve one additional QALY with the vaccination program.

The results show that vaccination is associated with an increased benefit in term of QALYs and increased costs

The calculated ICERs were 27 761, 99527 and 160827 SEK/QALY in cohorts of age 3,40 and 50 respectively. ( as shown in the table below)

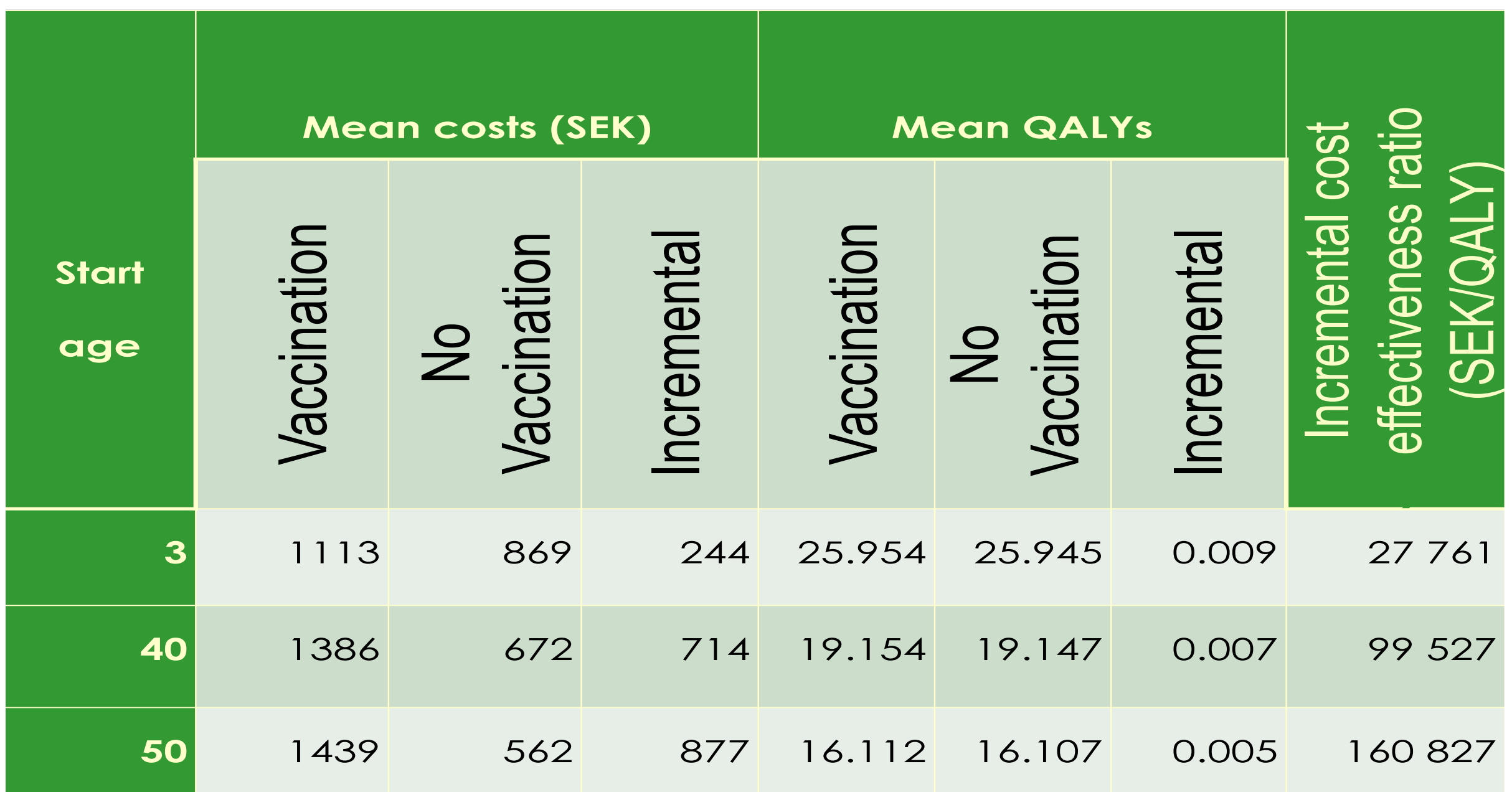

Cost effectiveness results of base case analysis

\section{Sensitivity analysis}

The one-way sensitivity analyses showed that the results are robust when varying different parameters.

A probabilistic sensitivity analysis was performed for different willingness to pay threshold.

Probabilistic results shows a high probability of cost-effectiveness at low thresholds (as shown in the graph):

Confirming the cost-effectiveness of the vaccination program.

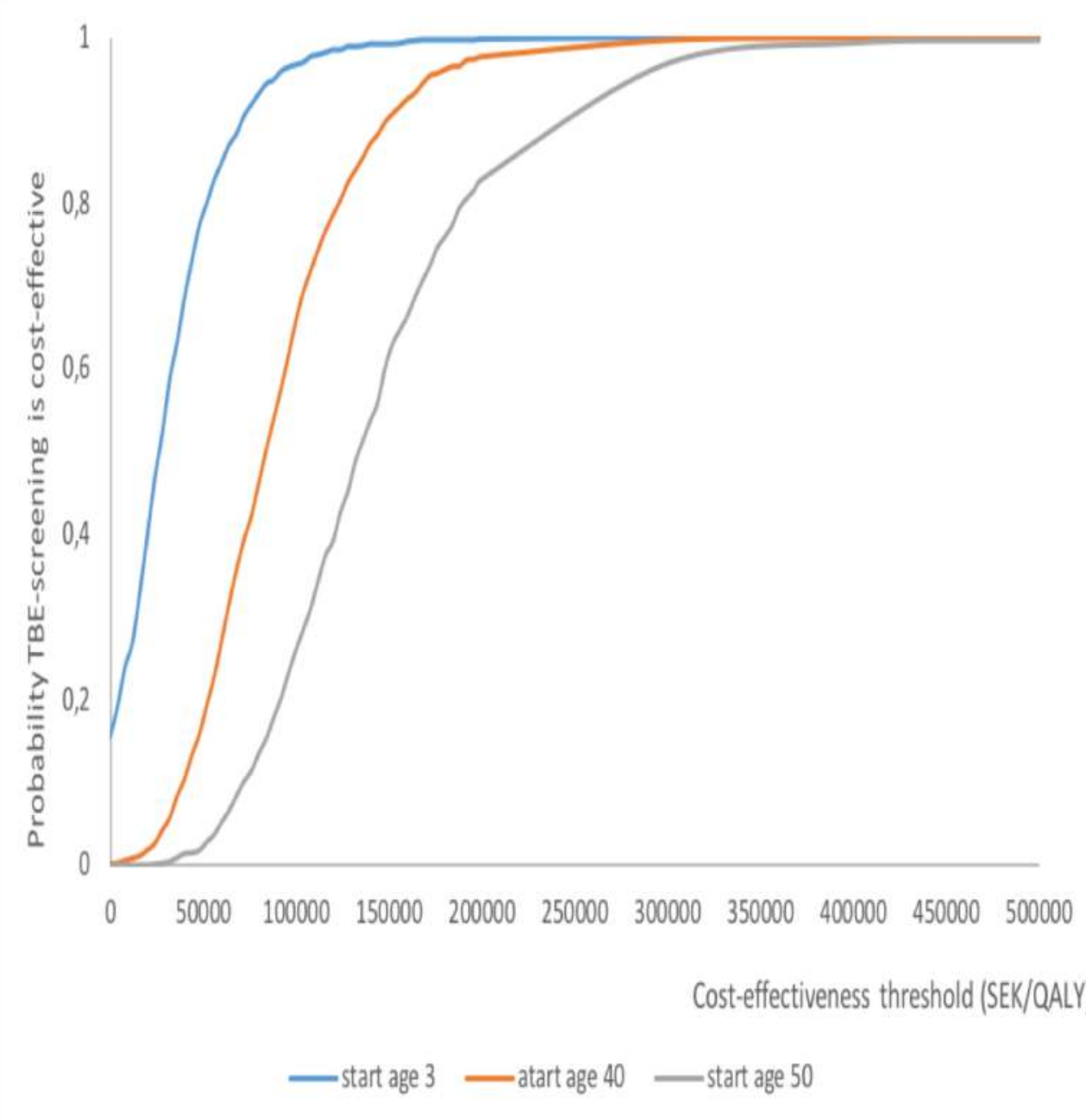

Cost effectiveness acceptability curves for the TBEvaccination program at different starting age.

\section{Conclusion}

This study shows that publiclyfinanced TBE vaccination has a relatively low ICER and costeffective mainly in the children cohort.

Therefore, we favor subsidizing the TBE vaccine since young age in Stockholm County.

${ }^{*}$ Corresponding author,

mail: jad.shedrawy@ki.se, Karolinska Institutet 